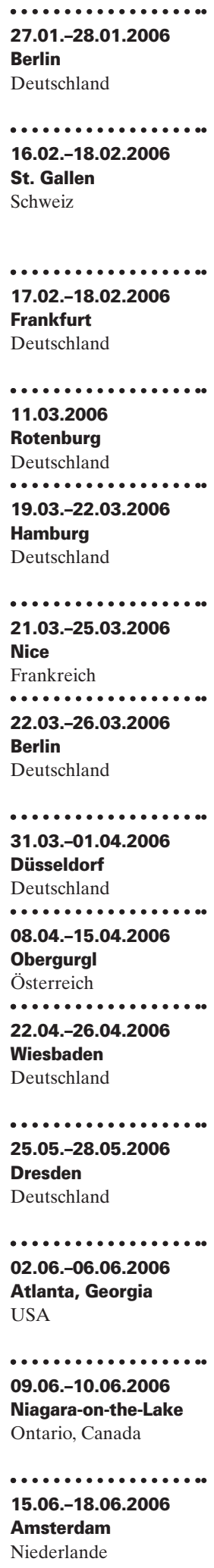

\section{Onko Update}

\section{International Conference}

Cancer Prevention 2006

(formerly: Tumor Prevention and Genetics)

Advances in Molecular and Clinical Aspects of Cancer Prevention

\section{Gynäkologie und Geburtshilfe Frankfurt}

\section{Rotenburger MAMMA-SYMPOSIUM}

Aktueller Stand, Entwicklungen und Perspektiven in der Diagnostik und Therapie beim Mammakarzinom

\section{Transplantation \\ 22. Meeting of the EBMT Nurses Group}

32. Annual Meeting of the European Group for Blood and Marrow

5. Meeting of the EBMT Data Management Group

\section{European Breast Cancer Conference}

27. Deutscher Krebskongress

6. Düsseldorfer Brustkrebs-Konferenz

6. Duesseldorf Breast Cancer Conference

1. Winter-Fortbildungstagung für Hämatologie und Onkologie

112. Kongress der Deutschen Gesellschaft für Innere Medizin

12. Jahreskongress der Deutschen Gesellschaft für Radioonkologie (DEGRO)

\section{Asco Annual Meeting}

9. International Conference on Long-Term Complications of Treatment of Children \& Adolescents for Cancer

11. Congress of the European Hematology Association (EHA)
Auskunft: COCS - Congress Organisation

C. Schäfer, München

E-mail katrin.lehmann@cocs.de

www.cocs.de

Auskunft: Conference Secretariat «CAP 2006»,

St. Gallen Oncology Conferences, Mrs. Beatrice Nair,

CH-St. Gallen

E-mailinfo@oncoconferences.ch

www.oncoconferences.ch

•............................

Auskunft: COCS - Congress Organisation

C. Schäfer, München

E-mail katrin.lehmann@cocs.de

www.cocs.de

Auskunft: Prof. Dr. med. Harald Meden, Rotenburg

E-mail gynsekre@diako-online.de

www.diako-online.de/frauenklinik

wa.

Auskunft: EBMT $2006 \mathrm{c} / \mathrm{o}$ AKM Congress Service,

CH-Basel

E-mail info@akm.ch

www.akm.ch/ebmt2006

Auskunft: EBCC-5 Conference Secretariat, Brussels E-mail EBCC5@fecs.be

www.fecs.be

Auskunft: Kongress- und Kulturmanagement GmbH, Weimar

E-mail info@kongresskultur.de

www.krebskongress2006.de

Auskunft: AAK GmbH, Düsseldorf

E-mail info@aakongress.de

www.aakongress.de

Auskunft: Eva M. Schaup, A-Wien

E-maileva@schaup.com

Auskunft: Daniel M. Metzler

m:con, Mannheim

E-mail daniel.metzler@mcon-mannheim.de

www.dgim2006.de

Auskunft: Deutsche Gesellschaft für Radioonkologie e.V., Berlin

Tel. +493084419188

E-mail office@degro.org

•...................000000000000....

Auskunft: American Society of Clinical Oncology,

Alexandria, VA, USA

E-mail asco@asco.org

www.asco.org

Auskunft: Daniel Green, MD, Pediatrics, Roswell

Park Cancer Institute, Buffalo, NY

Tel. (716) 845-2334, Fax (716) 845-8003

E-mail daniel.green@roswellpark.org

Auskunft: Eurocongres Conference Management,

NL-Amsterdam

Tel. +31 20679 3411, Fax +31206737306

E-mail: eha@eurocongres.com

www.eurocongres.com/eha2006/

\title{
KARGER
}


16.06.-17.06.2006

Dresden

Deutschland

\subsection{6.-01.07.2006}

Duisburg

Deutschland

\section{0. . . . . . . . .}

Mainz

Deutschland

\subsection{0 .000 \\ 14.07.-15.07.2006}

München

Deutschland

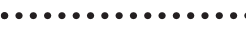

01.09.-02.09.2006

Kaiserslautern

Deutschland

\section{.................}

07.09.-09.09.2006

Salzburg

Österreich

\subsection{6}

Berlin

Deutschland

•................

29.09.-03.10.2006

Istanbul

Turkey

\section{..................}

04.11.-08.11.2006

Leipzig

Deutschland

\section{[................}

09.12.-12.12.2006

Orlando, Florida

USA

\section{$06.10-10.10 .2007$}

Basel

Schweiz
UPDATE Hämatologie / Onkologie 2006

UPDATE Hämatologie / Onkologie 2006

3. Jahreskongress Forum für Gastroenterologie

\section{UPDATE Hämatologie / Onkologie 2006}

178. Tagung der Mittelrheinischen Gesellschaft für Gynäkologie und Geburtshilfe

23. Jahrestagung der Österreichischen Gesellschaft für Senologie

56. Kongress der Deutschen Gesellschaft für Gynäkologie und Geburtshilfe

\section{ESMO 2006}

31. ESMO Congress

Gemeinsame Jahrestagung der Deutschen, Österreichischen und Schweizerischen Gesellschaften für Hämatologie und Onkologie

American Society for Hematology (ASH):

48. Annual Meeting

Gemeinsame Jahrestagung der Deutschen, Österreichischen und Schweizerischen Gesellschaften für Hämatologie und Onkologie
Auskunft: Interplan AG, München

E-mail t.hohenester@interplan.de

www.interplan.de/reg/update

Auskunft: Interplan AG, München

E-mail t.hohenester@interplan.de

www.interplan.de/reg/update

Auskunft: COCS - Congress Organisation

C. Schäfer, München

E-mail sandra.heussner@cocs.de

www.cocs.de

Auskunft: Interplan AG, München

E-mail t.hohenester@interplan.de

www.interplan.de/reg/update

Auskunft: COCS - Congress Organisation

C. Schäfer, München

E-mail katrin.lehmann@cocs.de

www.cocs.de

( Congress Organisation Thom

Wiese GmbH, Berlin

E-mail info@ctw-congress.de

www.ctw-congress.de/oegs

Auskunft: AAK GmbH, Düsseldorf

E-mailinfo@aakongress.de

www.aakongress.de

Auskunft: ESMO Head Office,

CH-Viganello-Lugano

E-mail congress@esmo.org

www.esmo.org

Auskunft: Hämatologie-Onkologie 2006

c/o AKM Congress Service, CH-Basel

E-mail info@akm.ch

www.akm.ch/onkologie-haematologie07

Auskunft: ASH Headquarters, Washington D.C.

USA

E-mail ash@hematology.org

www.hematology.org

Auskunft: Hämatologie-Onkologie 2006

c/o AKM Congress Service, CH-Basel

E-mailinfo@akm.ch

www.akm.ch/haematologie-onkologie06 